Revue internationale P.M.E.

Économie et gestion de la petite et moyenne entreprise

\title{
Les PME et l'intégration mondiale des systèmes de transport
}

\section{Yvon Bigras}

Volume 9, numéro 1, 1996

URI : https://id.erudit.org/iderudit/1008252ar

DOI : https://doi.org/10.7202/1008252ar

Aller au sommaire du numéro

\section{Éditeur(s)}

Presses de l’Université du Québec

\section{ISSN}

0776-5436 (imprimé)

1918-9699 (numérique)

Découvrir la revue

Citer cet article

Bigras, Y. (1996). Les PME et l'intégration mondiale des systèmes de transport Revue internationale P.M.E., 9(1), 21-40. https://doi.org/10.7202/1008252ar

\section{Résumé de l'article}

Le phénomène de la mondialisation de I'économie ne pourrait se produire sans une intégration de plus en plus poussée des systèmes logistiques et de transport à l'échelle mondiale. Pour évoluer sur ces marchés sans frontière, les entreprises doivent en effet compter sur des systèmes de transport efficaces et flexibles. Elles doivent respecter des standards de plus en plus élevés en ce qui concerne les délais, la fiabilité et le coût d'acheminement des produits. Dans cet article, nous faisons d'abord ressortir les quatre caractéristiques

fondamentales de cette intégration des systèmes de transport: le service autour du monde, l'intermodalité et l'amodalité, les changements dans les réseaux de transport et la concentration dans l'industrie du transport. Après avoir analysé les raisons expliquant cette intégration de même que ses limites, nous étudierons l'impact de cette intégration sur les PME, en insistant particulièrement sur le cas des PME québécoises. Notre analyse mettra en évidence la complexité accrue des décisions logistiques que doivent prendre les PME. Comme elles n’ont pas toujours les ressources nécessaires pour faire face à cette situation, elles doivent miser sur un partenariat logistique avec des prestataires pouvant leur offrir un soutien complet et adapté à leurs besoins.
Ce document est protégé par la loi sur le droit d'auteur. L’utilisation des services d’Érudit (y compris la reproduction) est assujettie à sa politique d'utilisation que vous pouvez consulter en ligne.

https://apropos.erudit.org/fr/usagers/politique-dutilisation/ 


\title{
Les PME et l'intégration mondiale des systèmes de transport
}

Yvon BIGRAS

Université du Québec à Trois-Rivières

\author{
MOTS CLÉS
}

\section{Transport-PME-Logistique-Mondialisation \\ Réseau-Déréglementation-Distribution Approvisionnement}

\begin{abstract}
RÉSUMÉ
Le phénomène de la mondialisation de l'économie ne pourrait se produire sans une intégration de plus en plus poussée des systèmes logistiques et de transport à l'échelle mondiale. Pour évoluer sur ces marchés sans frontière, les entreprises doivent en effet compter sur des systèmes de transport efficaces et flexibles. Elles doivent respecter des standards de plus en plus élevés en ce qui concerne les délais, la fiabilité et le coût d'acheminement des produits. Dans cet article, nous faisons d'abord ressortir les quatre caractéristiques fondamentales de cette intégration des systèmes de transport: le service autour du monde, l'intermodalité et l'amodalité, les changements dans les réseaux de transport et la concentration dans l'industrie du transport. Après avoir analysé les raisons expliquant cette intégration de même que ses limites, nous étudierons l'impact de cette intégration sur les PME, en insistant particulièrement sur le cas des PME québécoises. Notre analyse mettra en évidence la complexité accrue des décisions logistiques que doivent prendre les PME. Comme elles n'ont pas toujours les ressources nécessaires pour faire face à cette situation, elles doivent miser sur un partenariat logistique avec des prestataires pouvant leur offrir un soutien complet et adapté à leurs besoins.
\end{abstract}

\section{L'AUTEUR}

Yvon Bigras est professeur d'économique au Département des sciences de la gestion et de l'économie de I'Université du Québec à Trois-Rivières et membre du Groupe de recherche en économique et gestion des PME (GREPME). Adresse: GREPME, Université du Québec à Trois-Rivières, C.P. 500, Trois-Rivières (Québec), G9A 5H7. 


\begin{abstract}
The globalization of the world economy could not take place without a growing integration of transportation and logistics systems at a world-wide level. To compete in open markets, firms need efficient and flexible transportation systems. When moving freight, they must cope with higher and higher standards in terms of delay, reliability and cost. In this article we first define the four characteristics of an integrated transportation system: around the world door-to-door service, intermodalism, new transportation networks and concentration in the transportation industry. After an analysis of the reasons for this integration and its limits, we look at the impact on small and medium-sized enterprises (SMEs), with a special emphasis on Quebec's SMEs. The increasing complexity of logistical decisions is the major finding of this analysis. As SMEs do not have all the skill and resources to cope with this situation, they must develop strategic alliances with firms that can fulfil their specific needs for transportation and logistics services.
\end{abstract}

\title{
RESUMEN
}

El fenómeno de la mundializacion de la economía no podría producirse sin una integración cada vez más a la vanguardia de los sistemas logísticos y de transporte a escala mundial. En efecto, para evolucionar en mercados desprovistos de fronteras, las empresas deben contar con sistemas de transporte eficaces y flexibles. Deben respetar estándares cada vez más elevados a nivel de plazos, fiabilidad y costos de despacho de productos. En este artículo, hacemos resaltar en primer lugar las cuatro características fundamentales de la integración de los sistemas de transporte: el servicio alrededor del mundo, la intermodalidad, los cambios en las redes de transporte y la concentración de la industria del transporte. Después de haber analizado tanto las razones que explican esta integración así como sus limitaciones, estudiaremos su impacto en la PyME, insistiendo particularmente en el caso de las PyMEs quebequences. En nuestro análisis se destaca principalmente la complejidad creciente de las decisiones a nivel logístico que la pequeña y mediana empresa debe confrontar. Considerando que dichas empresa no cuentan siempre con los recursos necesarios para hacer frente a esta situación, éstas deben entonces contar con alianzas de tipo logístico cuyos contribuyentes sean capaces de ofrecerles un soporte íntegro y adaptado a sus necesidades especificas. 


\section{Introduction}

Les transformations qui remettent présentement en question les rapports économiques entre les nations, telles la spécialisation des économies, la libéralisation des échanges commerciaux, la mondialisation de la sous-traitance ou la montée de nouvelles puissances économiques, n'auraient pu se produire sans une intégration de plus en plus poussée des systèmes logistiques et de transport à l'échelle mondiale. Pour évoluer sur ces marchés sans frontière qui caractérisent de plus en plus l'économie mondiale, les entreprises doivent en effet compter sur des systèmes de transport efficaces, flexibles et fiables.

L'augmentation des exigences des clients a obligé les transporteurs à développer des approches qui éliminent les pertes de temps occasionnées par les opérations de manutention, de transmission de documents et de transition d'un mode à l'autre. Ces exigences se sont d'ailleurs affirmées avec le développement de modes de gestion axés sur la minimisation du niveau des inventaires et le recours au juste-à-temps qui permet de mieux synchroniser le cycle approvisionnement - production-distribution. Les entreprises impliquées dans une transaction internationale doivent donc respecter des standards de plus en plus élevés pour ce qui est des délais, de la fiabilité et du coût d'acheminement des produits. Elles ne se contentent plus d'acheter un service de transport entre deux points : elles demandent de plus en plus une prestation logistique intégrée pouvant aller jusqu'à la gestion des stocks, l'emballage et l'étiquetage.

Le phénomène d'intégration mondiale des systèmes de transport, qui répond à ces nouvelles exigences logistiques, n'aurait par ailleurs pu apparaître sans l'apport de certaines innovations technologiques (conteneurisation, interconnexion des réseaux informatiques, satellites de communication, etc.). La libéralisation des réglementations nationales et internationales des transports était un autre préalable au développement de services répondant aux nouvelles exigences des entreprises engagées dans le commerce international.

Le présent article a pour objectif d'évaluer dans quelle mesure les systèmes logistiques et de transport intégrés qui se sont développés à l'échelle mondiale sont susceptibles de répondre aux besoins particuliers des PME. Cette intégration ne se fait d'ailleurs pas sans quelques heurts et il sera d'abord nécessaire d'identifier ses caractéristiques, les raisons de cette intégration et ses limites. Malgré une certaine convergence et une tendance vers l'intermodalité, on notera des différences notables entre les modes de transport, entre certains segments de marchés ou entre les pays. Le débat sur la propriété étrangère des entreprises de transport illustre bien le genre de question qui n'a pas encore reçu de réponse définitive.

Ce nouvel environnement, qui découle de la mondialisation des économies et de l'intégration mondiale des systèmes de transport, pose par ailleurs de 
sérieux problèmes pour l'entreprise et pour la PME en particulier, en même temps qu'il lui offre de nouvelles possibilités. C'est ce que nous allons examiner de façon générale pour l'ensemble des PME, tout en insistant sur la situation des PME québécoises dans leur environnement nord-américain. En conclusion, on fera ressortir certaines conditions pouvant faciliter l'utilisation maximale par les PME des possibilités offertes par ce nouvel environnement.

\section{Intégration mondiale des systèmes de transport}

\subsection{Caractéristiques de cette intégration}

\subsubsection{Service autour du monde}

L'intégration des systèmes de transport se situe à deux niveaux : géographique et modes de transport. On a vu apparaître au cours des quinze dernières années des services de transport «autour du monde» assurés par des entreprises intégrées qui utilisaient plusieurs modes de transport. L'exemple le plus complet de ce type de service est donné par les transporteurs intégrés, comme FedEx, TNT, UPS, DHL, Emery. Ces entreprises, qu'on dit intégrées parce qu'elles sont intégrées verticalement et livrent leurs colis en se servant uniquement de leurs équipements, offrent des services «porte-à-porte » à la grandeur de la planète. Cette caractéristique de service mondial n'est cependant pas l'apanage des transporteurs intégrés. Les transporteurs aériens ou maritimes offrent de tels services en s'alliant à des transporteurs routiers locaux. En Amérique du Nord ou en Europe, la même exigence s'impose aux transporteurs routiers qui doivent, à défaut de couvrir l'ensemble de ces territoires, s'associer pour offrir un service «porte-à-porte» vers n'importe quelle destination.

Ce type de service répond aux besoins nouveaux des entreprises qui préfèrent acheter en une seule fois leurs services logistiques. Ces utilisateurs ont en effet pris conscience de la lourdeur et du coût associés au recours à plusieurs prestataires logistiques. Dans une étude réalisée par KPMG Peat Marwick Stevenson et Kellog (Bowland, 1992) sur les besoins des expéditeurs canadiens, on apprenait que ceux-ci plaçaient au quatrième rang les services "porte-à-porte », soit immédiatement après les tarifs de transport, la fiabilité du service et le temps total de transport. Dans le cas du transport international, il est encore plus important de pouvoir compter sur ce type de services, surtout pour une PME qui ne peut disposer d'un large réseau de distribution.

\subsubsection{Intermodalité et amodalité}

L'intégration mondiale des systèmes de transport se caractérise également par la disparition des frontières entre modes de transport. On parle d'intermodalité 
dans la mesure où les marchandises sont acheminées en combinant plusieurs modes de transport. Mais on tend de plus en plus vers l'amodalité, un concept proposé par Colin (1989), qui fait référence à la capacité du gestionnaire de la chaîne logistique de s'affranchir des modes de transport. Pour l'expéditeur qui achète un service logistique complet, il lui importe peu de savoir quel mode de transport sera utilisé.

En raison de ces changements, le transporteur est de plus en plus amené à élargir la gamme de services qu'il offre, soit directement ou par l'intermédiaire d'une alliance avec des transporteurs des autres modes. Le rapport annuel 1994 de l'Office national des transports du Canada (ONTC, 1995) donne plusieurs exemples de fusions et d'alliances entre transporteurs ferroviaires nord-américains et transporteurs routiers et maritimes qui ont été conclues pour favoriser l'établissement de services multimodaux intégrés. Un rapport de l'OCDE (1992) fait la même constatation pour l'Europe.

Les agencements intermodaux utilisés pour assurer des services «porte-à-porte autour du monde » efficaces sont par ailleurs très variés, voire surprenants. Par exemple, les biens à haute valeur-tels les appareils électroniques - qui sont exportés de l'Asie vers l'Amérique du Nord traversent parfois l'océan Pacifique en bateau avant de continuer leur course vers la côte est américaine par avion. Cela permet de ramener de 30 à 15 jours leur temps de transit, pour la moitié du coût d'un acheminement tout aérien (Dahm, 1992).

\subsubsection{Les changements dans les réseaux de transport}

Les transporteurs ont dû réorganiser leurs réseaux afin de desservir efficacement, donc de plus en plus rapidement et au moindre coût, des marchés origine-destination de plus en plus variés. La réponse principale de l'industrie a été de généraliser l'utilisation des réseaux en étoile (hub-and-spoke). Ces réseaux sont constitués de points pivots (aéroports, ports, centres de tri, etc.) de grande capacité situés au cœur des principaux marchés et entre lesquels les transporteurs effectuent des liaisons fréquentes, avec des équipements de grande taille et sur de grandes distances. Ces points centraux ou plaques tournantes (hubs) sont alimentés en passagers ou en marchandises, pour fins de consolidation, par un ensemble de liaisons régionales (spokes) vers des points secondaires. Grâce à la concentration des flux obtenue, ce type de réseaux permet des bénéfices appréciables sur les tronçons de longue distance reliant les points centraux.

Dès 1975, le transporteur routier américain Roadway utilisait un tel système sur une petite échelle. Mais ce sont les transporteurs intégrés dans le secteur des messageries qui ont été les premiers à organiser systématiquement 
l'ensemble de leurs opérations sur ce modèle, ce qui leur assure des économies considérables de champ et de réseau. Ces types de réseaux sont particulièrement populaires dans le transport aérien des passagers et des marchandises en Amérique du Nord et ailleurs (l'aéroport Schiphol d'Amsterdam est une immense plaque tournante pour KLM), dans le transport maritime, dans le transport des charges partielles par camion (le groupage routier) en Amérique du Nord et, de plus en plus, en Europe.

Si ces réseaux en étoile et l'utilisation de «plate-forme» peuvent être considérés comme une caractéristique des systèmes de transport intégrés à l'échelle mondiale, il faut quand même souligner que l'on assiste à un certain retour des liens plus directs. C'est le cas dans le transport aérien où l'on veut ainsi éviter de passer par des aéroports plaque tournante congestionnés. De la même façon, on voit de plus en plus les grandes surfaces dans le commerce de détail être approvisionnées par des livraisons directes aux points de vente, évitant ainsi les manutentions dans des entrepôts ou des centres de tri et de consolidation.

\subsubsection{Concentration dans l'industrie du transport}

La nécessité de gérer des techniques de transport mixtes, la multiplicité des échanges d'informations entre un nombre croissant de partenaires, la demande de services logistiques connexes au transport sont des facteurs qui poussent en faveur de la concentration ou, à tout le moins, en faveur d'alliances entre prestataires logistiques. Les PME de transport routier sont des opérateurs unimodaux insérés dans des chaînes qu'ils ne contrôlent pas. Face à eux, on retrouve de grandes entreprises avec des bureaux et des entrepôts situés à la grandeur d'un continent sinon de la planète et qui disposent de systèmes avancés d'information et de télécommunications. C'est le cas, par exemple, des trois grands de l'industrie nord-américaine du transport routier des charges partielles sur de longues distances qui possèdent plus de 600 terminus en Amérique du Nord. Leur position dominante sur le marché - ils détenaient $64 \%$ du marché en 1991 (Schulz, 1992) - a été acquise à la suite de l'organisation de leurs opérations sur le modèle des réseaux en étoile.

On assiste également à une concentration grandissante dans le transport aérien; et encore une fois, les réseaux en étoile sont au cœur de cette réorganisation. Dans ce cas, la concentration prend souvent la forme d'alliance entre transporteurs nationaux qui peut alors aller de la prise de participation majoritaire au simple partage des dénominations et des codes de vol. Plusieurs experts pensent que le secteur des transports aériens est voué dans un proche avenir à une concentration telle qu'il ne subsistera plus que cinq ou six coalitions de compagnies accaparant $90 \%$ du marché mondial. On retrouve un 
peu la même situation dans le transport des conteneurs où le trafic mondial est dominé par 15 mégatransporteurs (Commission d'examen de la Loi sur les transports nationaux, 1993). Ces derniers devront en plus établir des alliances entre eux s'ils veulent survivre, selon les termes employés par la Mitsui OSK Lines, dans son rapport annuel cité par l'Office national des transports (1995). Notons par ailleurs que cette concentration n'implique pas nécessairement l'absence de concurrence entre ces mégatransporteurs et une situation défavorable pour les utilisateurs. Il s'agit simplement d'une caractéristique de l'intégration mondiale des systèmes de transport.

Il faut par ailleurs souligner que le mouvement de concentration ne connaît pas la même ampleur dans tous les secteurs des transports. En même temps qu'on assiste à la concentration du transport aérien intercontinental, le marché des petits transporteurs régionaux demeure attrayant. Dans le transport ferroviaire aux États-Unis, les compagnies ferroviaires d'intérêt local accaparent maintenant $26 \%$ du réseau ferroviaire (ONT, 1995). Le secteur du camionnage voit aussi des petits transporteurs régionaux ou spécialisés bien se tirer d'affaires.

Cependant, aux économies d'utilisation de réseau (network utilization), de configuration de réseaux (network configuration) ou de champ (economies of scope) qui exercent de fortes pressions pour une concentration accrue dans plusieurs secteurs (Bigras, 1993), il faut ajouter un autre phénomène qui va dans le même sens et qui prend de l'ampleur. Il s'agit de la volonté des acheteurs de services de transport de réduire le nombre de leurs fournisseurs. Toutes les études récentes confirment que les entreprises ont réduit le nombre de transporteurs et de prestataires logistiques avec lesquels elles font affaires. On demande en conséquence à ceux que l'on a choisi d'offrir une gamme de services plus large, ce que peuvent faire des mégatransporteurs ou des transporteurs faisant partie d'un réseau de prestataires suffisamment large.

\subsection{Les raisons de cette intégration}

\subsubsection{Les nouvelles exigences logistiques}

Une première raison expliquant l'émergence de systèmes intégrés de transport est reliée à la demande de transport qui s'est internationalisée et complexifiée. Ces changements découlent directement de la globalisation économique tant dans la distribution des produits que dans leur production. On ne discutera pas en détail des divers phénomènes qui marquent cette globalisation, mais on doit certes souligner l'impact sur les exigences logistiques du passage d'une économie de masse à une «économie de la singularité » (Colin, 1989). La segmentation de plus en plus poussée des marchés qui caractérise cette économie 
pousse l'entreprise à étendre son aire géographique afin de compenser l'étroitesse des marchés. La grande fugacité des produits de consommation impose également une réduction du temps de distribution pour réduire le risque d'erreur de marketing. Au regard de la production, deux phénomènes, qui se conjuguent même parfois, sont déterminants : l'internationalisation de la sous-traitance et la gestion de production en flux tendus avec des̉ approvisionnements de type juste-à-temps.

L'ensemble de ces phénomènes requièrent des prestations de transport de plus en plus raffinées. Elles doivent en particulier répondre aux deux exigences de fluidité dans l'écoulement des marchandises et dans la continuité de la chaîne logistique (Colin, 1989). Les délais de livraison doivent non seulement être raccourcis, mais être également d'une fiabilité absolue. La distribution du produit de consommation, par exemple, se trouve au bout du canal de distribution et, contrairement à la production, il est alors impossible de réparer une erreur. Même dans la production, le recours au juste-à-temps limite la possibilité d'erreur dans la chaîne de transport. Le concept de qualité prend alors tout son sens. Il ne faut pas pour autant occulter l'importance des coûts dans ce contexte. La compétitivité des entreprises sur des marchés de plus en plus éloignés et diffus suppose également que ces prestations de service puissent se faire à un coût qui permette de vaincre cette distance.

\subsubsection{Le désengagement de l'État}

Au moment même où apparaissaient ces nouvelles exigences logistiques, on assistait au début d'un désengagement graduel de l'État dans le domaine du transport. Ce mouvement a d'abord pris la forme d'un assouplissement, voire d'une élimination dans certains cas, de la réglementation économique. Essentiellement, la déréglementation économique élimine les contrôles sur les prix de transport et libéralise l'accès à l'industrie. L'adoption du Airline Deregulation Act of 1978 aux États-Unis a marqué le début de ce mouvement, lequel s'est généralisé à tous les modes de transport aux États-Unis et dans une grande mesure au Canada, et s'étend maintenant ailleurs dans le monde. Le mouvement de privatisation des entreprises de transport étatiques s'est par ailleurs amplifié et il va également dans le sens de la déréglementation. Wheatcroft (1992) signalait il y a quelques années qu'une quarantaine de compagnies aériennes dans autant de pays étaient engagées dans un processus de privatisation. Le mouvement de déréglementation et de privatisation n'est, par ailleurs, pas indépendant de la mutation des exigences logistiques. Dans l'industrie du transport terrestre (routier et ferroviaire) en Amérique du Nord, la déréglementation semble avoir directement stimulé le développement de nouveaux services et de nouvelles pratiques logistiques, mouvements qui sont en fait interdépendants. 
Sur le plan international, la situation est plus complexe. La tarification des services aériens a été grandement assouplie, ce qui a eu pour effet d'atténuer la distinction entre vols réguliers et vols nolisés et de permettre une plus grande concurrence entre ces deux types de services. La promulgation aux États-Unis du US Shipping Act of 1984 a, de son côté, grandement diminué le pouvoir des conférences maritimes qui doivent subir une concurrence grandissante de navires indépendants (Brooks, 1992). On assiste par ailleurs à une ouverture des marchés de transport entre pays et à l'intérieur des pays. Ainsi, en 1997, le transport aérien dans la CEE sera complètement ouvert à tous les pays membres. L'Accord de libre-échange nord-américain (ALENA) prévoit également, au bout de dix ans, une ouverture complète des marchés transfrontaliers terrestres et la possibilité pour des investisseurs étrangers d'être propriétaires à part entière de transporteurs domestiques. Des discussions entre le Canada et les États-Unis ont même permis d'aller au-delà de l'accord pour permettre le cabotage au regard des équipements de transport routier. Cependant, cette entente, non encore officielle, ne touche pas pour l'instant les chauffeurs, ce qui en limite la portée.

Des négociations entre le Canada et les États-Unis ont, en outre, permis de conclure un accord de "ciel ouvert» (open skies) le 24 février 1995. Cet accord lève une grande partie des restrictions sur les voyages aériens entre le Canada et les États-Unis, tant sur le plan de la détermination des tarifs que sur celui de l'ouverture des marchés. Il faut toutefois noter que cet accord ne permet pas le cabotage, ce qui veut dire que les transporteurs d'un pays n'auront pas le droit de transporter des marchandises ou des voyageurs entre deux points dans l'autre pays.

Cette ouverture des marchés de transport entre pays et à l'intérieur des pays soulève actuellement la question de la propriété étrangère dans le secteur du transport ; il s'agit d'un enjeu majeur dans la réorganisation des systèmes de transport mondiaux. En libéralisant l'accès au marché domestique et en autorisant la propriété étrangère, cela permet à des entreprises étrangères de posséder une filiale dans le pays. C'est ce que font les transporteurs intégrés en acquérant ou en démarrant leur filiale dans chaque pays. Ils auraient évidemment eu de grandes difficultés à mettre leur système sur pied en présence d'une réglementation limitant la propriété étrangère. C'est également ce qui se passe dans le transport terrestre en Amérique du Nord où les compagnies ferroviaires canadiennes ont fait l'acquisition de plusieurs entreprises américaines. Dans le transport aérien, la question fera l'objet d'un débat acerbe, car, d'une part, il y a de fortes pressions pour une concentration de l'industrie en cinq ou six mégatransporteurs mondiaux et, d'autre part, les susceptibilités nationales défendent les porte-étendards aériens nationaux. 


\subsubsection{Les innovations technologiques}

L'intégration mondiale des systèmes de transport n'aurait finalement pu se réaliser en l'absence de certaines innovations technologiques. La première de ces innovations est le conteneur qui est essentiel pour un service "porte-àporte» autour du monde. Ce moyen permet d'éliminer les ruptures de charge tout en protégeant la marchandise, en particulier, contre le vol. Il s'agit là de conditions nécessaires pour assurer la fluidité et la continuité des flux à des coûts raisonnables. Elle a également permis une diminution importante des coûts de transport en diminuant les temps morts associés au chargement et au déchargement des marchandises. La révolution du conteneur ne s'est donc pas limitée à augmenter la productivité des navires et des ports : elle a imposé une restructuration complète du transport maritime de ligne et du transport en général en permettant l'intégration intermodale.

Le développement d'outils informatiques permettant une meilleure gestion des flux d'informations a également ouvert la porte à une offre de services de plus en plus complexes. Ces outils sont utilisés à l'interne (systèmes informatisés de répartition et d'entretien des équipements, par exemple); mais c'est dans les communications avec l'environnement externe (fournisseurs, clients, douanes, etc.) par l'échange de données informatisées (EDI) qu'ils sont appelés à jouer un rôle déterminant dans la création d'un avantage concurrentiel. Les exemples les plus spectaculaires des possibilités offertes par ces outils sont les systèmes de réservation des compagnies aériennes.

En même temps qu'ils sont de formidables atouts de vente, ces outils permettent de gérer la complexité qui découle des réseaux en étoile avec la souplesse nécessaire pour s'ajuster aux variations brusques de la demande. On comprend alors que ce système puisse assurer à un transporteur un avantage concurrentiel décisif. De plus, en raison des économies d'échelle considérables reliées à ces réseaux, les systèmes de réservation constituent une barrière à l'entrée favorisant la formation des mégatransporteurs ou alliances en voie de s'imposer sur l'industrie. Une évolution semblable se produit actuellement dans le transport routier des charges partielles sur de longues distances, où les systèmes de repérage des expéditions en temps réel avec l'aide de satellites sont un facteur jouant en faveur d'une concentration accrue. La baisse des coûts de ces technologies risque cependant de limiter cet effet dans la mesure où elles seront à la portée de tous les transporteurs. Cette situation comporte en revanche de nombreux avantages pour les utilisateurs qui se voient offrir de nouveaux services à des coûts abordables. 


\subsection{Les limites de l'intégration}

Malgré les forces favorisant l'intégration des transports, la concentration et la polarisation engendrée par les réseaux en étoile, la réalité est beaucoup plus complexe qu'il n'y paraît à première vue. Ainsi, il existe encore de vastes segments de l'industrie du transport qui semblent à l'abri de ces mouvements centripètes. On a précédemment souligné la persistance de petites entreprises de transport dans plusieurs secteurs de l'industrie. Dans certains cas, il peut s'agir des conséquences de reliquats réglementaires, mais dans de nombreux autres, il s'agit tout simplement d'une organisation plus efficace de l'industrie. Mais il y a d'autres freins au développement de l'intégration mondiale des transports telle que nous l'avons caractérisée. D'abord, l'existence de services «autour du monde» ne doit pas nous faire oublier qu'une part importante des flux de transport sont locaux et nationaux. Par exemple, si l'on considère le camionnage pour compte d'autrui au Canada, $69 \%$ du volume transporté est de nature intraprovinciale, $17 \%$ est interprovincial et $14 \%$ est international (Statistique Canada, 1993). Les données sur le camionnage pour compte propre ne sont pas disponibles, mais on sait que la proportion de flux intraprovinciaux y est encore plus importante. L'expansion de l'intermodalité n'est pas non plus généralisée. Dans les importations et les exportations entre le Canada et les États-Unis, le camion occupe la plus grande part du marché avec $75,8 \%$ et $62,7 \%$ respectivement. Le transport intermodal est, quant à lui, limité à 3,6\% et à $8,8 \%$. On peut aussi ajouter que le conteneur, qui est à la base des systèmes intermodaux, n'est pas sans connaître certains ratés : opérations de manutention et d'arrimage non appropriées, problèmes de normalisation, réglementations archaïques, trafic de drogues, etc. (Raffa, 1992).

Par ailleurs, l'organisation des réseaux de transport en étoile se heurte à certaines limites. La principale concerne la congestion qui découle d'un tel système. On connaît ces problèmes dans le transport aérien des passagers aux États-Unis. En effet, pour profiter des économies reliées à ces réseaux, il faut rassembler dans un aéroport principal des passagers qui proviennent de divers points de départ. Pour minimiser les temps de correspondance, on cherche alors à synchroniser les arrivées et les départs. Il s'ensuit des vagues successives de vols qui arrivent et partent en même temps et créent des heures de pointe très coûteuses sur le plan des ressources humaines et matérielles. On commence alors à assister à un retour des vols directs à partir des villes intermédiaires qui ne sont pas des plaques tournantes. Évidemment, cette tendance pourrait constituer un frein à la concentration dans la mesure où un plus petit transporteur peut exploiter une telle niche sans être pénalisé par sa taille.

Nous pourrions certes discuter longtemps des perspectives d'évolution des transports à l'échelle mondiale. Les quatre caractéristiques, que nous 
avons présentées pour définir le concept d'intégration mondiale des transports, nous semblent tout de même dominantes pour l'instant. Évidemment, cela n'exclut pas des mouvements opposés à ces tendances de fond sur certains segments de marché, et la libéralisation de cette industrie ouvre la porte aux ajustements qui correspondent le mieux aux besoins des expéditeurs.

\section{PME et choix logistiques}

Les changements dans les systèmes de transport sont en bonne partie une réponse à la mondialisation de l'économie et, à ce titre, ils permettent aux entreprises d'étendre leurs activités à l'échelle mondiale. Mais les conséquences pour les entreprises ne sont pas nécessairement uniformes et elles peuvent varier selon leur taille. On doit alors s'interroger sur les avantages et les inconvénients de cette intégration mondiale des systèmes de transport pour les PME.

Nous avons relevé quatre effets principaux de cette intégration sur les entreprises utilisatrices de services de transport. Ce sont la simplification des opérations logistiques, la complexité accrue des décisions logistiques, les nouvelles relations avec les fournisseurs de services logistiques et la création de nouveaux pôles logistiques. Ces effets seront analysés du point de vue des PME en faisant ressortir les occasions créées ou les défis supplémentaires que cela pose pour ces entreprises.

Par ailleurs, pour illustrer cette analyse, nous allons nous concentrer sur les conséquences que cette intégration entraîne pour les PME québécoises et canadiennes. Ces entreprises évoluent dans un contexte nord-américain où les systèmes de transport du Canada et des États-Unis sont en voie de fusion, ce qui représente jusqu'à un certain point un microcosme de l'intégration en cours sur le plan mondial. Nous pourrons également faire référence au transport outremer, mais nous insisterons sur le transport entre les deux pays, puisqu'il s'agit du commerce le plus important au monde entre deux pays et les États-Unis représentent plus de $80 \%$ du commerce extérieur du Canada.

Pour saisir l'influence sur les PME de ces changements dans les transports, nous utiliserons trois principales sources de données. Pour le Québec, nous utiliserons les résultats de deux enquêtes que nous avons menées pour le ministère des Transports du Québec. La première portait sur les activités de logistique et de transport des entreprises expéditrices dans le contexte de la

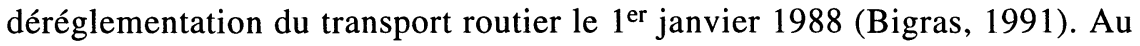
total, nous avons recueilli des informations par sondage postal auprès de 665 établissements, en plus de réaliser 23 entrevues. Pour notre analyse, nous nous sommes concentré sur les 482 PME qui figuraient parmi ces établis- 
sements, soit 341 PME manufacturières (1 - 199 employés) et 141 PME du secteur du commerce de gros (1 - 49 employés). Pour compléter notre analyse et la mettre à jour, nous avons utilisé les informations recueillies auprès d'une quinzaine d'experts de la logistique dans le cadre d'une enquête sur les nouvelles pratiques logistiques au Québec (Bigras, 1995). Nous avons finalement utilisé les données compilées par l'Office national des transports du Canada dans deux de ses enquêtes annuelles sur l'industrie du transport (ONT, 1992 et 1995).

\subsection{Simplification des opérations}

La caractéristique la plus frappante du développement des transports terrestres en Amérique du Nord au cours des dernières années est la diversification des services. Les expéditeurs ont maintenant le choix entre une gamme de produits de configuration et de prix différents. Que ce soit des services de livraison «juste-à-temps », de «livraison le lendemain » ou des possibilités de rabais en acceptant des livraisons à tel moment de la journée, les expéditeurs ont pu obtenir des transporteurs le type de service adapté à leurs besoins. De façon générale, cette diversification des services simplifie les opérations des entreprises qui peuvent trouver, voire négocier, le type de services qui leur convient.

On peut s'en convaincre en regardant, dans le tableau 1, les niveaux de satisfaction très élevés que nous avons obtenus auprès des PME québécoises. En effet, 27,5\% des PME étaient «très satisfaites » et 66,0\% étaient «assez satisfaites » des services offerts par les transporteurs routiers avec lesquels elles faisaient affaires. Le tableau 2 permet en outre de constater que ces PME notaient dans $55,5 \%$ des cas qu'il y avait eu une amélioration de la qualité des services de transport de charges complètes extraprovinciales (c'est-à-dire dont la destination finale était à l'extérieur du Québec) depuis la libéralisation de ce secteur. Seulement 7,7\% des PME relevaient une détérioration de la qualité des services. Pour les charges partielles, la situation était semblable avec des taux respectifs de $46,7 \%$ et $7,2 \%$. On remarque par ailleurs la même amélioration chez les utilisateurs de services ferroviaires (ONT, 1992), et il s'agit d'une situation qui ne s'est pas démentie depuis.

TABLEAU 1

Satisfaction par rapport aux services offerts par les transporteurs routiers (\% des PME)

Très satisfait 27,5

Assez satisfait 66,0

Peu satisfait

6,0

Pas du tout satisfait

0,6 
TABLEAU 2

Changements dans la qualité des services de camionnage Flux extraprovinciaux (\% des PME)

\begin{tabular}{lcc}
\hline & Charges complètes & Charges partielles \\
\hline Amélioration notable & 20,7 & 15,4 \\
Amélioration mineure & 34,8 & 31,3 \\
Aucun changement & 36,8 & 46,1 \\
Détérioration mineure & 4,3 & 5,7 \\
Détérioration majeure & 3,4 & 1,5 \\
\hline
\end{tabular}

Cette amélioration des services s'est également produite alors que les taux unitaires de transport subissaient de fortes pressions à la baisse. Ainsi, au regard des flux extraprovinciaux de charges complètes par camion, $42,5 \%$ des PME ont bénéficié d'une baisse ou de la stabilité des taux de transport entre le début de 1988 et le mois de juin 1991. Seulement 14,3\% des établissements ont subi des hausses de tarifs supérieures à $10 \%$ pendant ces 42 mois. La situation était un peu moins favorable aux expéditeurs sur le marché des charges partielles, tout en demeurant fort avantageuse avec seulement 20,0\% des PME subissant une hausse des prix de transport de plus de $10 \%$ pendant ces 42 mois. On peut d'ailleurs signaler que ces conditions tarifaires avantageuses s'appliquaient tant aux PME qu'aux grandes entreprises. Ce qui vient apaiser les craintes de ceux qui croyaient que la déréglementation allait créer une situation désavantageuse pour les petits expéditeurs. La même constatation s'applique aux expéditeurs en régions périphériques qui ont le plus bénéficié des baisses de tarifs et de l'amélioration des services. Encore là, il s'agit d'une situation qui n'a pas changé au cours de la récession de 1991-1992. Avec la reprise de l'économie et de l'activité de transport au cours des deux dernières années, les prix du transport ont remonté, mais la concurrence très vive continue d'exercer des pressions pour faire baisser les prix.

L'amélioration concerne tous les aspects des services, que ce soit les temps de transit, la fréquence des cueillettes, la disponibilité des équipements, le suivi de l'expédition, etc. L'amélioration de la qualité des services intermodaux a également été notée, quoique le camion continue de dominer outrageusement le trafic continental nord-américain. Les transporteurs se doivent maintenant d'offrir des services complets en Amérique du Nord et ils ont rationalisé leurs opérations en établissant des réseaux en étoile et des itinéraires triangulaires, ou en s'alliant à d'autres transporteurs, par exemple. L'élimination des permis a ainsi ouvert la porte à une rationalisation des opérations qui se mesure principalement par la diminution des retours à vide.

Si les PME ont pu profiter de ces avantages, c'est à cause de l'intensification de la concurrence. C'est le cas, par exemple, dans les transports ferro- 
viaires où le recours aux contrats confidentiels s'est généralisé. Dans le transport routier, l'enquête de 1991 indique que la majorité des PME québécoises considèrent que le nombre de transporteurs offrant leurs services a augmenté. Sur le marché des charges complètes transfrontalières, seulement 15,5\% des PME sont d'avis que la concurrence a diminué et, sur le marché des charges partielles, ce pourcentage n'est que de $7,9 \%$. Ces résultats peuvent surprendre dans la mesure où l'on assiste à un mouvement de concentration dans l'industrie. Ce mouvement, que l'on observe en Amérique du Nord, a en fait été plus que compensé par l'extension des marchés desservis par les transporteurs existants. Globalement, il y a moins de transporteurs, mais en général pour les utilisateurs, il y a plus d'offreurs de services étant donné que plusieurs transporteurs, dont les transporteurs régionaux de plus petite taille, ont étendu leur aire de marché au-delà des limites stipulées dans leurs anciens permis.

\subsection{Complexité accrue des décisions}

La diversification des services et l'intensification de la concurrence, bien qu'elles simplifient les opérations des entreprises, rendent plus complexes leurs choix stratégiques ou opérationnels. Que ce soit la décision d'avoir leurs propres camions ou de consolider quelques expéditions, les options sont beaucoup plus nombreuses qu'auparavant. Comme les transporteurs essaient de plus en plus de moduler leurs prix en fonction de plusieurs variables, même l'heure de la journée où se fera la cueillette devient un facteur à considérer dans la prise en compte de décision; dans un environnement réglementé et protégé de la concurrence, le chargeur n'avait bien souvent pas de choix à cet égard.

Pour obtenir ces résultats, les entreprises ont cependant dû négocier avec les transporteurs routiers et ferroviaires. Ainsi, pour le transport routier en $1991,56 \%$ des expéditeurs canadiens déclarent avoir réussi à négocier à la baisse les tarifs d'abord proposés (Office national des transports, 1992). Mais il n'y a pas que les tarifs qui sont contestés, les prestations de services font également l'objet de négociations. Lorsque celles-ci n'aboutissent pas, la concurrence offre une porte de sortie aux expéditeurs. Une donnée de l'enquête auprès des PME québécoises, que l'on retrouve au tableau 3, illustre de façon éloquente cette situation nouvelle. En effet, 76,5\% des PME déclarent avoir changé certains des transporteurs avec lesquels elles faisaient affaires régulièrement. La raison principale pour justifier cette «rupture»: les tarifs dans $51,3 \%$ des cas et l'amélioration du service dans $43,1 \%$ des cas. Cela implique également que les PME doivent évaluer fréquemment la performance de leurs transporteurs. On constate en effet que 45,5\% d'entre elles disent évaluer «souvent» leur performance, $41,8 \%$ le font «à l'occasion », 11,6\% «rarement» et $1,0 \%$ «jamais». 
TABleau 3

PME ayant changé certains de leurs transporteurs et raisons invoquées (\% des PME)

Pourcentage des PME ayant changé

76,5

Principale raison invoquée

Tarifs

51,3

Services

43,1

Autres raisons

Les occasions d'affaires créées par la diversité des services offerts et les exigences accrues des marchés ouverts ont également provoqué une véritable révolution des pratiques logistiques dans l'entreprise. Du début de 1988 à juin 1991, le tableau 4 révèle que $60,8 \%$ des PME ont modifié leur façon de gérer leurs stocks. Les principales raisons invoquées pour expliquer ces changements sont les suivantes: diminuer le niveau des stocks $(67,4 \%)$, augmenter la rotation des stocks $(47,5 \%)$, s'adapter à un système de production « juste-à-temps » $(32,5 \%)$. On note évidemment que la pratique du juste-àtemps n'est plus marginale et qu'elle commence à se répandre chez les PME. Notre enquête auprès d'experts de la logistique confirme d'ailleurs l'effet prépondérant de cette pratique sur la logistique et le transport au Québec et en Amérique du Nord (Bigras, 1995).

TABlEAU 4

Modifications de la façon de gérer les stocks

(\% des PME)

Oui

60,8

Non 39,2

\subsection{Nouvelles relations avec les fournisseurs de services logistiques}

On a déjà souligné que la concurrence entre les transporteurs avait amené les expéditeurs à négocier avec les transporteurs, tant sur les taux de transport que sur la nature des services fournis. Si les expéditeurs n'obtiennent pas ce qu'ils croient raisonnables, ils peuvent maintenant changer de transporteurs, alors que dans un environnement réglementé, ils devaient accepter le service des transporteurs ayant l'autorisation de faire des affaires sur leur territoire. Les transporteurs maintenaient avec leur clientèle des liens personnels plutôt paternalistes. On confiait pendant des années ses expéditions au même transporteur sans vraiment évaluer sa performance qui, de toute façon, variait bien peu d'un transporteur à l'autre. L'ouverture de ce secteur à la concurrence 
donne maintenant une liberté de choix à l'utilisateur qui ne se prive pas d'en faire usage pour obtenir des conditions plus avantageuses. On remarque par ailleurs une évolution vers un modèle de partenariat logistique qui tend à remplacer la stratégie qui consistait, dans la phase d'ajustement postdéréglementation, à confronter systématiquement les transporteurs les uns aux autres au regard des prix demandés.

L'évolution implique évidemment que le nombre de transporteurs avec lesquels il sera possible de transiger diminuera. L'Office national des transports (1992) note en effet que près de quatre expéditeurs sur dix déclarent avoir réduit le nombre de transporteurs avec qui ils font affaires. Cela correspond à la stratégie d'un nombre grandissant de transporteurs qui tentent d'obtenir une part accrue des marchandises d'un client donné en lui offrant des services à valeur ajoutée d'entreposage et de planification logistique. La guerre tarifaire a été très coûteuse pour l'industrie et elle a amené des taux de rendement anémiques après la déréglementation. La voie d'une plus grande collaboration entre transporteurs et expéditeurs semble maintenant devoir s'imposer. La question de la capacité excédentaire de l'industrie est évidemment cruciale pour l'évolution sur ce point. Dans le cas du transport aérien, par exemple, il s'agit de la question de l'heure. Mais la situation dans le transport routier est relativement différente, d'une part, en raison de la plus grande souplesse d'ajustement de cette capacité et, d'autre part, parce que le camionnage ne subit pas autant que le transport aérien l'ingérence des gouvernements qui veulent défendre les avions portant les couleurs nationales.

Pour les PME, cette collaboration avec les transporteurs se fait cependant dans des conditions nouvelles. En raison de l'intégration et de la concentration, la PME doit de plus en plus souvent négocier avec un transporteur qui est une entreprise de grande taille ou à tout le moins relié à un groupe de grande taille. La PME dont le volume d'expéditions est limité risque peut-être d'être défavorisée dans ce contexte. On en a un exemple dans le transport ferroviaire au Canada où le nombre de contrats confidentiels a progressé en moyenne au rythme de $61 \%$ par année depuis 1988 . On calcule maintenant que, en moyenne, $70 \%$ du trafic des expéditeurs est acheminé suivant de telles ententes. On constate toutefois que cette pratique est beaucoup plus répandue chez les grands expéditeurs (plus de dix millions de dollars de frais de transport), puisque $96 \%$ d'entre eux y ont eu recours, alors que seulement $31 \%$ des petits expéditeurs (moins de un million de dollars de frais de transport) ont réussi à négocier de telles ententes (ONT, 1992).

Cependant, les taux élevés de satisfaction notés plus haut dans le camionnage et l'évolution très favorable des taux de transport semblent démontrer que les PME n'ont pas été pénalisées jusqu'à présent. La concurrence entre des entreprises de transport plus grandes demeure encore très vive, 
et les PME dans les grands centres ou en régions ont accès à de meilleurs services que jamais. L'utilisation de courtiers et d'intermédiaires, en hausse particulièrement auprès des petits expéditeurs (ONT, 1992), est aussi un facteur qui permet aux PME d'obtenir de meilleurs taux, car ces intermédiaires font du groupage et négocient directement avec les transporteurs.

\subsection{Nouveaux pôles logistiques}

L'intégration mondiale des systèmes de transport, tout en permettant de vaincre la distance en offrant des services efficaces autour du monde, restructure l'espace économique. La constitution de réseaux en étoile y joue certainement un rôle déterminant. Ces réseaux reposent sur une hiérarchisation de l'espace selon qu'une localité est ou n'est pas un point central et selon le niveau de ce point central (principal, secondaire ou local). La qualité des services de transport à partir d'un point variera alors en fonction de sa situation dans le réseau. Un aéroport qui devient un aéroport pivot offrira une gamme de services directs vers un plus grand nombre de destinations qu'un aéroport positionné sur un rayon du réseau. À partir de ce dernier point, tous les déplacements impliqueront une escale et un itinéraire passant par un aéroport pivot.

Ce phénomène pourrait éventuellement influencer la localisation des entreprises, particulièrement les PME dont les volumes d'expéditions sont insuffisants pour organiser un transport adapté à leurs besoins. Cependant, il peut y avoir un coût associé à une localisation à un point central si celui-ci est dominé par un ou deux transporteurs, ce qui est souvent le cas. On a en effet calculé que les voyageurs aériens américains originaires d'un aéroport pivot doivent débourser environ $18,7 \%$ de plus lorsqu'un transporteur domine l'aéroport en question et $8,9 \%$ de plus lorsque deux transporteurs se partagent le marché (Secretary's Task Force, 1990). Le voyageur se trouve, par conséquent, captif des transporteurs opérant aux aéroports pivots pour l'ensemble de son itinéraire. Par contre, en raison des incertitudes concernant le développement des réseaux en étoile, ce qu'illustre le retour de liaisons directes entre des points secondaires, et la lenteur avec laquelle peuvent apparaître des effets sur la localisation des entreprises, il est sans doute trop tôt pour bien évaluer l'influence de ces développements sur la PME.

\section{Conclusion}

\section{Les défis pour la PME}

La mondialisation de l'économie est accompagnée par un mouvement d'intégration mondiale des systèmes de transport. Malgré des variantes locales et la persistance de segments de l'industrie relativement isolés, cette intégration 
modifie sensiblement l'environnement dans lequel évoluent les PME. Cela représente d'abord pour les PME la possibilité de simplifier leurs opérations grâce à une gamme de services très variés, de bonne qualité et à des coûts qui reflètent une vive concurrence.

Malgré tout, ce nouvel environnement soulève certaines interrogations. Les PME doivent faire des choix beaucoup plus complexes qu'auparavant et elles n'ont pas nécessairement toutes les compétences ni toutes les ressources pour structurer une chaîne logistique et de transport performante et compétitive. Elles se retrouvent de plus en plus face à de très grandes entreprises fournisseurs de services logistiques et de transport qui pourraient éventuellement détenir un pouvoir décisif sur la compétitivité des PME. Finalement, l'intégration mondiale des réseaux de transport pourra créer de nouveaux pôles logistiques qui pourraient rendre caduque la localisation de certaines PME.

Dans ce contexte, les PME doivent miser sur des alliances interentreprises auxquelles seront associées les entreprises fournisseurs de services logistiques et de transport. Ces alliances, telles que les définit Saget, sont précisément «un moyen pour acquérir au meilleur prix les ressources externes qui deviennent nécessaires pour assurer le développement de la PME» (Saget, 1992). Les PME doivent donc miser sur un partenariat logistique avec des prestataires pouvant leur offrir un soutien complet et adapté à leurs besoins. Or, au Québec du moins, on a toujours vu les transporteurs comme de simples fournisseurs de services sur demande, sans les associer à la planification de l'entreprise. Il est donc urgent de changer cette mentalité. Les transporteurs devront, de leur côté, offrir des services plus complets et s'allier avec d'autres prestataires logistiques pour offrir des services «porte-à-porte» autour du monde. Quant aux PME de transport, elles pourront survivre à la condition de bien cibler certaines niches de marché et de négocier des alliances leur permettant d'offrir des services de classe mondiale.

\section{Bibliographie}

BIGRAS, Y. (1991), Étude des effets de la déréglementation économique auprès des usagers de services de camionnage, Rapport présenté au ministère des Transports du Québec.

BIGRAS, Y. (1993), «La déréglementation de l'industrie du camionnage au Québec: de la théorie à la réalité », L'Actualité économique, vol. $69, \mathrm{n}^{\circ} 3$, septembre, p. 123-138.

BIGRAS, Y. (1995), La demande de transport de marchandises au Québec et dans ses régions: caractéristiques et perspectives, Rapport présenté au ministère des Transports du Québec. 
Bowland, J.M. (1992), Canadian Shippers' Needs Assessment, KPMG Peat Marwick Stevenson and Kellog, Rapport remis à la Commission d'examen de la Loi sur les transports nationaux.

BROOKS, M.R. (1992), «International competitiveness - Assessing and exploiting competitive advantage by Ocean container carriers ", Actes de la $27^{e}$ conférence du Canadian Transportation Research Forum, p. 343-354.

Colin, J. (1989), «Les nouveaux transporteurs», Logistiques Magazine, ${ }^{0}$ 35, février, p. 58-60.

COMMISSION D'EXAMEN DE LA LOI SUR LES TRANSPORTS NATIONAUX (1993), La concurrence dans les transports : regard sur la politique et la législation, Approvisionnements et Services Canada.

DAHM, D. (1992), "The ports of Seattle and Vancouver: some competitive differences ", Actes de la $27^{e}$ conférence du Canadian Transportation Research Forum, p. 331-342.

OFFICE NATIONAL DES TRANSPORTS DU CANADA (1992), Examen annuel 1991, Approvisionnements et Services Canada.

OFFICE NATIONAL DES TRANSPORTS DU CANADA (1995), Examen annuel 1994, Approvisionnements et Services Canada.

RAFFA, R. (1992), "Une face cachée de la révolution du conteneur », Actes de la $27^{e}$ conférence du Canadian Transportation Research Forum, p. 366-377.

SAGET, F. (1992), «Impact de la globalisation économique sur les PME: l'apprentissage des alliances », Texte préparé pour la Conférence sur les PME et la globalisation économique tenue à Montréal, OCDE.

SCHULZ, J.D. (1992), «Big Three LTL carriers find riches in diversification, regional units », Traffic World, 31 août, p. 17-22.

SECRETARY'S TASK FORCE ON COMPETITION IN THE U.S. DOMESTIC AIRLINE INDUSTRY (1990), Executive Summary, Washington, D.C., U.S. Departement of Transportation.

Statistique Canada (1993), Le camionnage au Canada 1990, $\mathrm{n}^{\circ}$ cat. 53-222, Ottawa.

WHEATCROFT, S. (1992), «Airlines : reaping the rewards of globalisation », Transport World, supplément de Transport, novembre-décembre, p. 1-2. 